\title{
BMJ Open Neutrophil-to-lymphocyte ratio as a predictor of mortality in intensive care unit patients: a retrospective analysis of the Medical Information Mart for Intensive Care III Database
}

Xie Wu, ${ }^{1}$ Qipeng Luo, ${ }^{2}$ Zhanhao Su, ${ }^{3}$ Yinan Li, ${ }^{1}$ Hongbai Wang, ${ }^{1}$ Qiao Liu, ${ }^{1}$ Su Yuan (D) , ${ }^{1}$ Fuxia Yan (D) ${ }^{1}$

To cite: Wu X, Luo Q, Su Z, et al. Neutrophil-to-lymphocyte ratio as a predictor of mortality in intensive care unit patients: a retrospective analysis of the Medical Information Mart for Intensive Care III Database. BMJ Open 2021;11:e053548. doi:10.1136/ bmjopen-2021-053548

- Prepublication history and additional supplemental material for this paper are available online. To view these files, please visit the journal online (http://dx.doi.org/10.1136/ bmjopen-2021-053548).

Received 19 May 2021 Accepted 26 October 2021

\section{Check for updates}

(c) Author(s) (or their employer(s)) 2021. Re-use permitted under CC BY-NC. No commercial re-use. See rights and permissions. Published by BMJ.

${ }^{1}$ Department of Anesthesiology, Chinese Academy of Medical Sciences and Peking Union Medical College Fuwai Hospital, Xicheng District, Beijing, China ${ }^{2}$ Department of Pain Medicine, Peking University Third Hospital, Beijing, China

${ }^{3}$ Department of Pediatric Cardiac Surgery, Chinese Academy of Medical Sciences and Peking Union Medical College Fuwai Hospital, Beijing, China

Correspondence to

Dr Fuxia Yan;

yanfuxia@sina.com

\section{ABSTRACT}

Objectives Identifying high-risk patients in the intensive care unit (ICU) is important given the high mortality rate. However, existing scoring systems lack easily accessible, low-cost and effective inflammatory markers. We aimed to identify inflammatory markers in routine blood tests to predict mortality in ICU patients and evaluate their predictive power.

Design Retrospective case-control study.

Setting Single secondary care centre.

Participants We analysed data from the Medical Information Mart for Intensive Care III database. A total of 21822 ICU patients were enrolled and divided into survival and death groups based on in-hospital mortality.

Primary and secondary outcome measures The predictive values of potential inflammatory markers were evaluated and compared using receiver operating characteristic curve analysis. After identifying the neutrophil-to-lymphocyte ratio (NLR) as having the best predictive ability, patients were redivided into low ( $\leq 1)$, medium (1-6) and high (>6) NLR groups. Univariate and multivariate logistic regression analyses were performed to evaluate the association between the NLR and mortality. The area under the curve (AUC), net reclassification improvement (NRI) and integrated discrimination improvement (IDI) were used to assess whether incorporating the NLR could improve the predictive power of existing scoring systems.

Results The NLR had the best predictive ability (AUC: 0.609; $\mathrm{p}<0.001)$. In-hospital mortality rates were significantly higher in the low (OR (OR): $2.09 ; 95 \% \mathrm{Cl} 1.64$ to 2.66) and high (OR $1.64 ; 95 \% \mathrm{Cl} 1.50$ to 1.80 ) NLR groups than in the medium NLR group. Adding the NLR to the Simplified Acute Physiology Score II improved the AUC from 0.789 to 0.798 , with an NRI and IDI of $16.64 \%$ and $0.27 \%$, respectively.

Conclusions The NLR predicted mortality in ICU patients well. Both low and high NLRs were associated with elevated mortality rates, including the NLR may improve the predictive power of the Simplified Acute Physiology Score II.

\section{BACKGROUND}

Patients admitted to the intensive care unit (ICU) are usually severely ill, with high mortality rates and high hospital costs. ${ }^{1}$
Strengths and limitations of this study

- This study included a large sample size and avoided selection bias by inclusion of all intensive care unit patients.

- This study noticed that the mortality rate was also elevated in patients with a low neutrophil-tolymphocyte ratio.

- The design was retrospective and important data may be missing; reasons for the missing data (especially those of neutrophil or lymphocyte counts) were challenging to determine based on the available information.

- The conclusions are qualitative rather than quantitative

Therefore, identifying patients with a high risk of mortality is essential. Existing scoring systems to predict the risk of mortality in the ICU, such as the Simplified Acute Physiology Score (SAPS) and Acute Physiology and Chronic Health Evaluation, ${ }^{2}$ do not include effective inflammatory markers. C reactive protein and procalcitonin concentrations are widely recognised as indicators of inflammation; however, routine testing is not always available for every ICU patient because of cost considerations, especially for patients without infectious complications. Thus, identifying low-cost, easily accessible and effective inflammatory markers may help predict mortality in ICU patients.

A blood examination is one of the routine tests conducted for every patient admitted to the ICU. In addition to total white blood cell (WBC) and differential counts, combined markers, such as the neutrophilto-lymphocyte ratio (NLR) and platelet-tolymphocyte ratio (PLR), have also attracted much attention in recent years. Numerous 
studies $^{3-11}$ have focused on the prognostic value of inflammatory markers in routine blood tests; however, the most sensitive indicator remains to be identified. More importantly, in clinical practice, we noted that some patients with a low NLR have a poor prognosis. However, when examining the literature, we found that although there are many studies on the NLR, ${ }^{3-11}$ most of them concluded that a high NLR was associated with a poor prognosis, but ignored the prognostic value of a low NLR. Therefore, we conducted this study to verify which indicator is the best inflammatory marker in routine blood tests and to assess its prognostic value for mortality in ICU patients.

\section{METHODS}

\section{Data sources}

Data for this study were obtained from the Medical Information Mart for Intensive Care III (MIMIC-III) database V.1.4 (https://mimic.physionet.org), which is a large, publicly available database comprising information on $>40000$ patients who were admitted to the critical care unit of Beth Israel Deaconess Medical Center. Restrictions apply to the availability of these data, which were used under license for this study. XW completed the Collaborative Institutional Training Initiative programme and was responsible for data extraction (certification number: 35931746).

\section{Participants}

All patients who were admitted to the ICU were included. The exclusion criteria were as follows: (1) patients who are not the first hospitalisation, (2) patients younger than 16 years old, (3) patients who had no blood routine test data within 24 hours of hospitalisation, (4) patients with abnormal values for key variables. Abnormal values in this study were defined as extreme outliers, that is WBC count $>400 \times 10^{9} / \mathrm{L}, \mathrm{NLR}>100$ and PLR $>8000$. Based on these inclusion and exclusion criteria, 21822 patients were finally enrolled for data analysis.

\section{Data extraction}

Data from the MIMIC-III database were extracted using structured query language within PostgreSQL (V.11.2, https://www.postgresql.org/). Demographic data, laboratory parameters, the clinical outcomes of patients and survival data were collected from all participants, including data on age; sex; ethnicity; ICU type; WBC, lymphocyte, neutrophil, and platelet counts; ICU and hospital lengths of stay; in-hospital mortality and 90-day and 1-year mortality. Severity at admission was measured using the SAPS II. Laboratory parameters were assessed during the first 24 hours after admission. The NLR and PLR were calculated by dividing the neutrophil or platelet count by the lymphocyte count. The SAPS II was automatically calculated in the database according to published scoring criteria. ${ }^{12}$ Extracted data were presented in comma-separated value files, linked by identifiers, and integrated into a table using Stata V.15.0 (Stata, Texas).

\section{Statistical analyses}

Statistical analyses were performed using Stata V.15.0 and MedCalc V.19.0.7 (MedCalc, Mariakerke, Belgium). Continuous variables are presented as medians with IQRs and were compared using the Wilcoxon rank sum test or Kruskal-Wallis test. Categorical variables are presented as frequencies with percentages and were compared using the Fisher's exact test or binomial probability test. Receiver operating characteristic curves were plotted to calculate the area under the curve (AUC) and were compared using the DeLong test. Optimal cut-off values for each inflammatory marker were determined using MedCalc V.19.0.7. Univariate and multivariate logistic regression analyses were performed to evaluate the prognostic value of the NLR for mortality. In the multivariate analysis, we adjusted for variables with a $\mathrm{p}$ value less than 0.2 in the univariate analysis or clinically significant, including age, sex, ethnicity, ICU type and the SAPS II. In addition to the traditional AUC, net reclassification improvement (NRI) and integrated discrimination improvement (IDI) were calculated to assess improvements in predictive power after adding the NLR. Subgroup analyses were performed to evaluate whether main diagnosis could influence the results. A $\mathrm{p}<0.05$ was considered statistically significant.

\section{Patient and public involvement}

Patients or the public were not involved in the design, or conduct, or reporting or dissemination plans of this research.

\section{RESULTS}

Between June 2001 and October 2012, a total of 38597 patients ( $\geq 16$ years) were admitted to the ICU. After the selection criteria were applied, 21822 patients were included in the final analysis. The flow diagram of this study is presented in figure 1 . There were no missing data except for age (missing for $0.2 \%$ of cases, $\mathrm{n}=42$ ), and it was missing at random, so we replaced it with the mean value. The median (IQR) age of these patients was 66.68 (52.76 to 79.55 ) years and $46.47 \%$ were women. The in-hospital mortality rate was $14.43 \%$, while the 90 -day and 1-year mortality rates were $20.78 \%$ and $28.57 \%$, respectively. The median (IQR) lengths of ICU and hospital stay were 2.08 (1.21-4.13) and 6.63 (3.79-11.79) days, respectively.

Based on the in-hospital mortality data, patients were divided into survival and death groups. The baseline characteristics and clinical data are shown in table 1 . The death group was older and had more women than the survival group. Compared with overall in-hospital mortality, the mortality rate in the medical ICU was significantly higher ( $14.43 \%$ vs $16.31 \%$, respectively). Blood examinations showed that the WBC count, neutrophil count, NLR and PLR were significantly higher, whereas the lymphocyte and platelet counts were significantly lower, in the death group.

The AUC for all inflammatory markers and their optimal cut-off values are shown in online supplemental 
58976 ICU patients

Excluded 37154 patients

- Re-admitted to the ICU: 12458

- < 16y: 7921

- Lack of blood routine test data:16659

- Abnormal values: 116

21822 patients for analysis

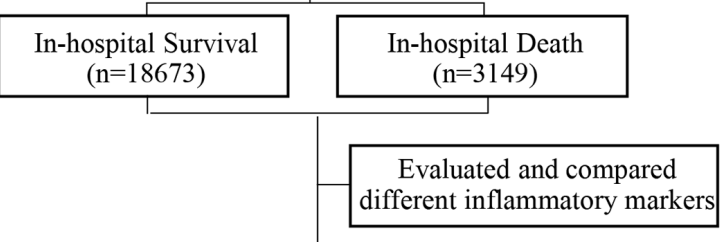

NLR had the best predictive ability

\begin{tabular}{|c|c|}
\cline { 2 - 2 } \multicolumn{1}{c|}{} & $\begin{array}{c}\text { Translated NLR into } \\
\text { categorical variable }\end{array}$ \\
\hline $\begin{array}{c}\text { Determine the association } \\
\text { between NLR and mortality }\end{array}$ \\
\hline
\end{tabular}

Figure 1 Study flow diagram in the present study. ICU, intensive care unit; NLR, neutrophil-to-lymphocyte ratio.

table 1 . The NLR had the greatest ability to predict in-hospital mortality (AUC: 0.609; $\mathrm{p}<0.001$ ). The in-hospital mortality rates for different NLRs are shown in online supplemental figure 1 . We found that both a high $(>6)$ and low $(\leq 1)$ NLR were associated with a higher mortality rate. Therefore, we selected the NLR as our best inflammatory marker, with cut-off values of 1 and 6 .

We further divided patients into three groups based on the NLR-low (NLR $\leq 1 ; \mathrm{n}=580)$, medium $(1<\mathrm{NLR} \leq 6$; $\mathrm{n}=10691)$ and high (NLR $>6 ; \mathrm{n}=10551)$ NLR groupsand compared the clinical outcomes (table 2). The baseline of the three groups is presented in online supplemental table 2. Compared with the medium NLR group, the low and high NLR groups were both significantly associated with a poor prognosis. Their in-hospital, 90-day and 1-year mortality rates were significantly higher, and the hospital and ICU stays were also significantly longer.

Table 3 presents the results of the logistic regression analyses for the association between the NLR and mortality. In the univariate analysis, the NLR was significantly associated with in-hospital, 90-day and 1-year mortality. Very high or low NLRs may both be associated with elevated mortality rates. Similar results were obtained in the multivariate analysis after adjusting for age, sex, ethnicity, ICU type and the SAPS II.

The predictive value of the NLR was evaluated by calculating the AUC, NRI and IDI. As shown in figure 2, the addition of the NLR to the SAPS II significantly improved the AUC from 0.789 (95\% CI 0.785 to 0.796$)$ to 0.798 (95\% CI 0.793 to $0.804 ; \mathrm{p}<0.001$, DeLong test). The NRI and IDI for the NLR in relation to the SAPS II were $16.64 \%(\mathrm{p}<0.001)$ and $0.27 \% \quad(\mathrm{p}<0.001)$, respectively.
We also performed a subgroup analysis based on main diagnosis (online supplemental table 3). The prognostic value of the NLR in the subgroups was similar to that of the total, except for patients with chronic liver disease.

\section{DISCUSSION}

The main findings of this study are as follows. The NLR had the best predictive ability for in-hospital mortality in ICU patients. Further analyses based on the NLR revealed that patients with a high or low NLR were more likely to have higher mortality rates and longer ICU and hospital stays. The addition of the NLR significantly improved the predictive power of the SAPS II, and the results of the subgroup analysis based on main diagnosis were consistent with the overall population.

The predictive value of the NLR has been widely studied, particularly in cardiovascular disease, ${ }^{58}$ infectious disease ${ }^{79}$ and cancer. ${ }^{1011} 13$ In this study, among the inflammatory indicators of the routine blood tests, NLR has the strongest ability to predict in-hospital death. NLR alone as a predictor has limited predictive power, and its AUC value is only 0.609 . This may be related to that the prognosis of ICU patients is affected by many other confounding factors. However, after translating NLR into categorical variable and adjusting for the confounding factors, NLR showed a good ability to predict ICU outcomes. In addition, most previous studies ${ }^{3-11} 1415$ have suggested that the higher the NLR, the worse the prognosis; however, other studies ${ }^{16}{ }^{17}$ have suggested that a low NLR is also associated with a poor prognosis. If, as in previous studies, we divided patients equally into 3-5 groups based on their NLR, we could draw the same conclusion that a high NLR is indicative of a poor prognosis. However, before analysis, we noted that patients with a low NLR also seemed to have a poor prognosis. Thus, we implemented a different grouping scheme and confirmed our hypothesis by further analysis. Indeed, this finding was in line with clinical experience: the prognosis is generally good when the clinical indicators are within the normal range, and values that are too high or low are more likely to be associated with a poor prognosis. Several studies ${ }^{1318}$ have suggested that the reason why an elevated NLR leads to a poor prognosis is mainly because of enhanced systemic inflammation and stress responses. However, the reason why a low NLR is associated with a poor prognosis remains unclear. We speculated that a decreased NLR may be due to a decrease in neutrophils that play a key role in the innate immune response, including directly killing pathogens by phagocytosis, releasing a variety of cytokines, and activating T cells, among other roles. ${ }^{19}$ Therefore, a reduction in circulating neutrophils can lower the body's response to microbial invasion. In addition, reduced circulating neutrophils can be ascribed to the increased neutrophil adhesion to the vascular endothelium, which can cause endothelial damage, leading to leucocyte aggregation and microvascular thrombosis. ${ }^{20}$ Thus, the compromise 
Table 1 Baseline characteristics according to survivors and death

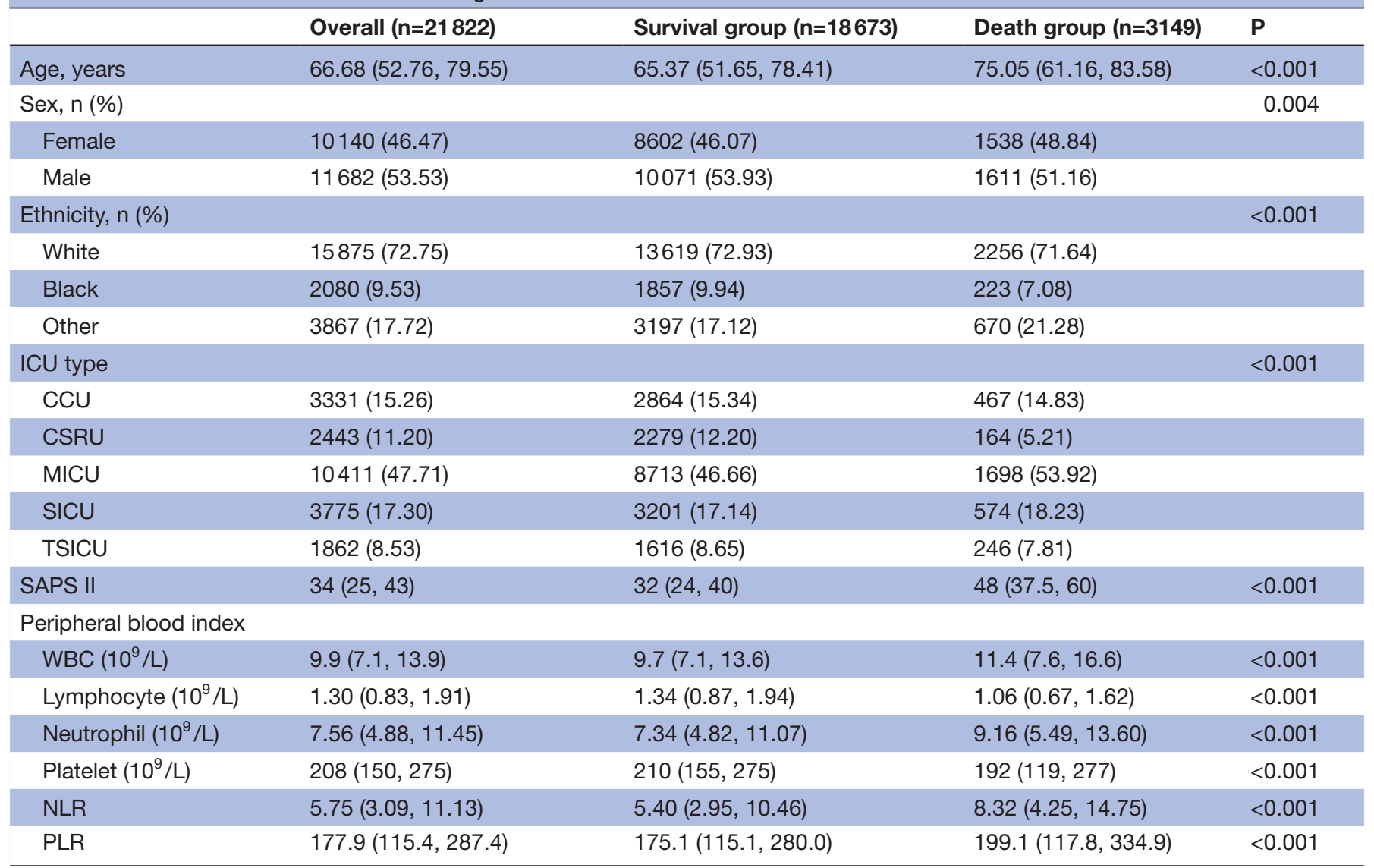

Data are presented as median and IQR or number and percentage.

CCU, coronary care unit; CSRU, cardiac surgery recovery units; ICU, intensive care unit; MICU, medical ICU; NLR, neutrophil-tolymphocyte ratio; PLR, platelet-to-lymphocyte ratio; SAPS, Simplified Acute Physiology Score; SICU, surgical ICU; TSICU, trauma surgical ICU; WBC, white blood cell.

of innate immunity and the increase in endothelial damage can collectively impair the prognosis of patients.

Many previous studies ${ }^{3-11} 1415$ have overlooked the possibility of a low NLR, leading to a poor prognosis, which may be due to several reasons. First, compared with the overall trend towards a high NLR correlating with a poor prognosis, the association between a low NLR and a poor prognosis may have been neglected due to the small number of patients. There were only 580 patients with an NLR $\leq 1$, which was $2.66 \%$ of the total population.
Second, the main outcome indicators may have influenced the conclusions. Previous studies, which have mostly focused on late death ( $\geq 5$ days), found that a high NLR can predict a poor prognosis. However, Riché et al ${ }^{17}$ reported that a low NLR is associated with an early death ( $<5$ days $)$, whereas a high NLR is associated with a late death. Duggal et $a l^{18}$ also suggested that an elevated NLR is a biomarker for an increased length of ICU stay. Therefore, based on previous studies that focused on late death, it is reasonable to conclude that a high NLR is associated

Table 2 Clinical outcomes of the ICU patients

\begin{tabular}{|c|c|c|c|c|c|}
\hline \multirow[b]{2}{*}{ Clinical outcomes } & \multirow[b]{2}{*}{ Overall $(n=21822)$} & \multicolumn{3}{|l|}{ NLR } & \multirow[b]{2}{*}{$\mathbf{P}$} \\
\hline & & $\leq 1(n=580)$ & $1-6(n=10691)$ & $>6(n=10551)$ & \\
\hline Hospital mortality, n (\%) & $3149(14.43)$ & $122(21.03)$ & 1009 (9.44) & $2018(19.13)$ & $<0.001$ \\
\hline 1-year mortality, n (\%) & $6234(28.57)$ & $211(36.38)$ & $2311(21.62)$ & $3712(35.18)$ & $<0.001$ \\
\hline ICU length of stay (days) & $2.08(1.21,4.13)$ & $2.04(1.08,4.38)$ & $1.96(1.13,3.46)$ & $2.38(1.33,5.04)$ & $<0.001$ \\
\hline
\end{tabular}

Data are presented as median and IQR or number and percentage.

ICU, intensive care unit; NLR, neutrophil-to-lymphocyte ratio. 
Table 3 Association between NLR and mortality

\begin{tabular}{|c|c|c|c|c|}
\hline \multirow[b]{2}{*}{ Exposure } & \multicolumn{2}{|l|}{ Non-adjusted } & \multicolumn{2}{|l|}{ Adjusted } \\
\hline & OR (95\% Cl) & $\mathbf{P}$ & OR $(95 \% \mathrm{Cl})$ & $\mathbf{P}$ \\
\hline \multicolumn{5}{|c|}{ In-hospital mortality } \\
\hline $1-6$ & 1 & & 1 & \\
\hline$>6$ & 2.27 (2.09 to 2.46 ) & $<0.001$ & 1.59 (1.46 to 1.74$)$ & $<0.001$ \\
\hline \multicolumn{5}{|c|}{ 90-day mortality } \\
\hline $1-6$ & 1 & & 1 & \\
\hline$>6$ & 2.08 (1.95 to 2.22 ) & $<0.001$ & 1.60 (1.48 to 1.43$)$ & $<0.001$ \\
\hline \multicolumn{5}{|c|}{ 1-year mortality } \\
\hline$\leq 1$ & 2.07 (1.74 to 2.47$)$ & $<0.001$ & 1.51 (1.23 to 1.86$)$ & $<0.001$ \\
\hline
\end{tabular}

Adjusted confounders: age, sex, ethnicity, ICU type and SAPS II.

ICU, intensive care unit; NLR, neutrophil-to-lymphocyte ratio; SAPS, Simplified Acute Physiology Score.

with increased mortality. However, in our study, around half of the in-hospital deaths (1512/3149; 48.02\%) occurred within 5 days; thus, our study indicated that a low NLR is also associated with increased mortality. Third, the study population may have influenced the conclusions. Several studies have been conducted in patients with specific diseases, ${ }^{113}$ and those excluded often had a low NLR. Our study focused on all ICU patients with no case selection.

Commonly used ICU prognosis score includes acute physiology and chronic health evaluation (APACHE), sequential organ failure assessment (SOFA), SAPS and so

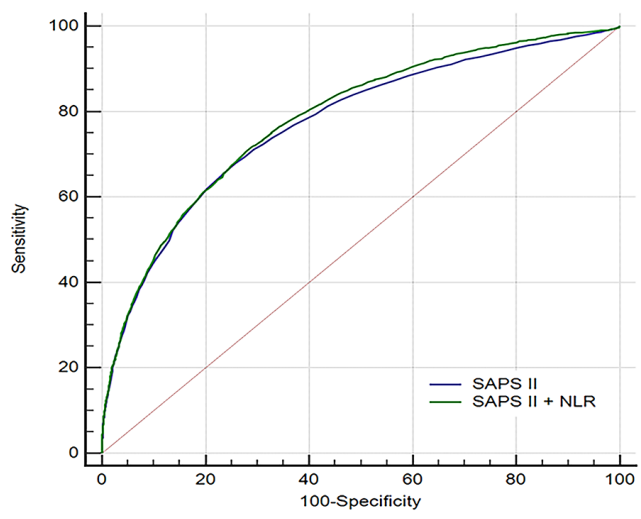

\begin{tabular}{|c|c|c|c|}
\hline \multirow{3}{*}{$\mathrm{AUC}$} & SAPS II & SAPSII+NLR & P-value \\
\hline & 0.789 & 0.798 & \multirow[t]{2}{*}{$<0.001$} \\
\hline & \multicolumn{2}{|c|}{ SAPS II vs. SAPSII+NLR } & \\
\hline NRI & \multicolumn{2}{|c|}{$16.64 \%$} & $<0.001$ \\
\hline IDI & \multicolumn{2}{|c|}{$0.27 \%$} & $<0.001$ \\
\hline
\end{tabular}

Figure 2 Receiver operating characteristic curves for the SAPS II and the SAPS II+NLR. AUC, area under the receiveroperating characteristic curve; IDI, integrated discrimination improvement; NLR, neutrophil-to-lymphocyte ratio; NRI, net reclassification improvement; SAPS, Simplified Acute Physiology Score. on. ${ }^{21}{ }^{22}$ In this study, the SAPS was chosen because of its lack of inflammatory indicators. Although the SAPS III has better predictive ability, ${ }^{23}$ there were too many missing values, because it requires collecting data within 1 hour after admission; therefore, we chose to use the SAPS II. As an easy-to-obtain, sensitive inflammatory indicator that does not increase the financial burden of patients, NLR has been reported by many previous studies and has high clinical significance. That is why we tried to add NLR to the SAPS II to evaluate whether it can increase its predictive performance. After adding NLR, the AUC of SAPS II increased from 0.789 to 0.798 . Although the AUC value increase is statistically different, the increase is very small. Therefore, in order to illustrate the clinical importance of NLR, we also calculated NRI and IDI, and the results indicated that the addition of NLR significantly improved the prediction ability, with an NRI of $16.64 \%$ and IDI of $0.27 \%$, respectively. What is more, some studies suggested that the PLR also had the ability to predict mortality ${ }^{24}$; therefore, we evaluated the predictive power of the PLR and found that, despite having some predictive ability, it was not as effective as the NLR. When we added the PLR to the SAPS II together with the NLR, the AUC value did not increase significantly; therefore, we did not incorporate the PLR into this model.

The major strengths of our study are the large sample size and the inclusion of all ICU patients without selection bias. Furthermore, we noticed that the mortality rate was also elevated in patients with a low NLR. More importantly, we found that adding the NLR to the SAPS II could improve its predictive power for ICU mortality, which is an important prompt for future scoring systems and may be of particular interest to critical care specialists.

There are also some limitations to this study. First, this was a retrospective study and some important data may be missing. Some patients were excluded because of 
missing neutrophil or lymphocyte data, and it was difficult to explore the reasons for missing data based on the information currently available. Second, the conclusions of this study were qualitative rather than quantitative. We can only infer that the addition of the NLR can improve the performance of the SAPS II because the NLR scores cannot be directly included in the SAPS II to construct a new scoring system, however, creating a new prognostic model is not a goal of this study, the main purpose of this study was to attract the attention of clinicians to be wary of abnormal NLR values. Finally, although we conducted a subgroup analysis of different diagnosis, in-depth analyses were not undertaken as it was not the aim of our study.

\section{CONCLUSIONS}

Of the inflammatory markers identified from routine blood tests, the NLR was the best predictor of ICU mortality. Abnormally high or low NLRs were associated with increased mortality. Finally, the addition of the NLR to the SAPS II can improve its predictive power for ICU mortality.

Acknowledgements The authors would like to thank the Medical Information Mart for Intensive Care III (MIMIC-III) database version 1.4 (https://mimic.physionet.org) for providing open access, and Editage (www.editage.com) for their writing support.

Contributors XW was the guarantor, and she was fully responsible for all stages of the study, including design, data extraction, statistical analysis and manuscript writing. FY and SY were involved in the design of the original protocol. QLu and $Y L$ participated in data curation and analyses. HW and QLi contributed to the discussion and interpretation of data. ZS helped to draft the final manuscript. All authors read approved the final manuscript.

Funding This work was supported by the National Nature Science Foundation of China, grant number 81870070 , and the Innovative Foundation for Graduate Students at Peking Union Medical College, grant number 2019-1002-78.

Disclaimer The views expressed in the manuscript are our own and not an official position of the institution or funder.

Competing interests None declared.

Patient consent for publication Not applicable.

Ethics approval Since MIMIC-III is a third-party, anonymised, publicly available database with pre-existing Institutional Review Board approval at BIDMC and MIT, we were exempted from obtaining approval from our institution.

Provenance and peer review Not commissioned; externally peer reviewed.

Data availability statement Data are available in a public, open access repository.

Supplemental material This content has been supplied by the author(s). It has not been vetted by BMJ Publishing Group Limited (BMJ) and may not have been peer-reviewed. Any opinions or recommendations discussed are solely those of the author(s) and are not endorsed by BMJ. BMJ disclaims all liability and responsibility arising from any reliance placed on the content. Where the content includes any translated material, BMJ does not warrant the accuracy and reliability of the translations (including but not limited to local regulations, clinical guidelines, terminology, drug names and drug dosages), and is not responsible for any error and/or omissions arising from translation and adaptation or otherwise.

Open access This is an open access article distributed in accordance with the Creative Commons Attribution Non Commercial (CC BY-NC 4.0) license, which permits others to distribute, remix, adapt, build upon this work non-commercially, and license their derivative works on different terms, provided the original work is properly cited, appropriate credit is given, any changes made indicated, and the use is non-commercial. See: http://creativecommons.org/licenses/by-nc/4.0/.

\section{ORCID iDs}

Su Yuan http://orcid.org/0000-0003-3924-7548
Fuxia Yan http://orcid.org/0000-0001-5054-0775

\section{REFERENCES}

1 Adhikari NKJ, Fowler RA, Bhagwanjee S, et al. Critical care and the global burden of critical illness in adults. Lancet 2010;376:1339-46.

2 Lemeshow S, Le Gall JR. Modeling the severity of illness of ICU patients. A systems update. JAMA 1994;272:1049-55.

3 Fest J, Ruiter TR, Groot Koerkamp B, et al. The neutrophilto-lymphocyte ratio is associated with mortality in the general population: the Rotterdam study. Eur J Epidemiol 2019;34:463-70.

4 Salciccioli JD, Marshall DC, Pimentel MAF, et al. The association between the neutrophil-to-lymphocyte ratio and mortality in critical illness: an observational cohort study. Crit Care 2015;19:13.

5 Sun H, Que J, Peng Y, et al. The neutrophil-lymphocyte ratio: A promising predictor of mortality in coronary care unit patients - A cohort study. Int Immunopharmacol 2019;74:105692.

6 Yoldas H, Karagoz I, Ogun MN, et al. Novel mortality markers for critically ill patients. J Intensive Care Med 2020;35:383-5.

7 Ye W, Chen X, Huang Y, et al. The association between neutrophilto-lymphocyte count ratio and mortality in septic patients: a retrospective analysis of the MIMIC-III database. J Thorac Dis 2020;12:1843-55.

$8 \mathrm{Kim} \mathrm{S}$, Eliot M, Koestler DC, et al. Association of neutrophil-tolymphocyte ratio with mortality and cardiovascular disease in the Jackson heart study and modification by the Duffy antigen variant. JAMA Cardiol 2018;3:455-62.

9 Russell CD, Parajuli A, Gale HJ, et al. The utility of peripheral blood leucocyte ratios as biomarkers in infectious diseases: a systematic review and meta-analysis. J Infect 2019;78:339-48.

10 Valero C, Zanoni DK, McGill MR, et al. Pretreatment peripheral blood leukocytes are independent predictors of survival in oral cavity cancer. Cancer 2020;126:994-1003.

11 Shi M, Zhao W, Zhou F, et al. Neutrophil or platelet-to-lymphocyte ratios in blood are associated with poor prognosis of pulmonary large cell neuroendocrine carcinoma. Transl Lung Cancer Res 2020;9:45-54.

12 Le Gall JR, Lemeshow S, Saulnier F. A new simplified acute physiology score (SAPS II) based on a European/North American multicenter study. JAMA 1993;270:2957-63.

13 Gemenetzis G, Bagante F, Griffin JF, et al. Neutrophil-to-lymphocyte ratio is a predictive marker for invasive malignancy in intraductal papillary mucinous neoplasms of the pancreas. Ann Surg 2017;266:339-45.

14 Karagoz I, Yoldas H. Platelet to lymphocyte and neutrophil to lymphocyte ratios as strong predictors of mortality in intensive care population. Rev Assoc Med Bras 2019;65:633-6.

15 Ham SY, Yoon HJ, Nam SB, et al. Prognostic value of neutrophil/ lymphocyte ratio and mean platelet volume/platelet ratio for 1-year mortality in critically ill patients. Sci Rep 2020;10:21513.

16 Hwang SY, Shin TG, Jo IJ, et al. Neutrophil-to-lymphocyte ratio as a prognostic marker in critically-ill septic patients. Am J Emerg Med 2017;35:234-9.

17 Riché F, Gayat E, Barthélémy R, et al. Reversal of neutrophil-tolymphocyte count ratio in early versus late death from septic shock. Crit Care 2015;19:439.

18 Duggal NA, Snelson C, Shaheen U, et al. Innate and adaptive immune dysregulation in critically ill ICU patients. Sci Rep 2018;8:10186.

19 Uhl B, Vadlau Y, Zuchtriegel G, et al. Aged neutrophils contribute to the first line of defense in the acute inflammatory response. Blood 2016;128:2327-37.

20 Entman ML, Youker K, Shappell SB, et al. Neutrophil adherence to isolated adult canine myocytes. Evidence for a CD18-dependent mechanism. J Clin Invest 1990;85:1497-506.

21 Sinto R, Suwarto S, Lie KC, et al. Prognostic accuracy of the quick Sequential Organ Failure Assessment (qSOFA)-lactate criteria for mortality in adults with suspected bacterial infection in the emergency department of a hospital with limited resources. Emerg Med J 2020;37:363-9.

22 Kalichsztein M, Nobre G, Kezen J, et al. Using outcome prediction tools in the ICU: performance of APACHE II and SAPS 2 scores in clinical patients. Crit Care 2006;10:P407.

23 Polzik P, Grøndal O, Tavenier J, et al. SuPAR correlates with mortality and clinical severity in patients with necrotizing soft-tissue infections: results from a prospective, observational cohort study. Sci Rep 2019;9:5098.

24 Shen Y, Huang X, Zhang W. Platelet-to-lymphocyte ratio as a prognostic predictor of mortality for sepsis: interaction effect with disease severity-a retrospective study. BMJ Open 2019;9:e22896. 